\title{
Primeiro relato de larvas de Eustrongylides sp. (Nematoda: Dioctophymatidae) em Hoplias malabaricus (Characiformes: Erythrinidae) no Estado de Rondônia, Amazônia Ocidental, Brasil
}

First report of Eustrongylides sp. larvae (Nematoda: Dioctophymatidae) in Hoplias malabaricus (Characiformes: Erythrinidae) in Rondônia State, Western Amazon, Brazil

Primer relato de larvas de Eustrongylides sp. (Nematodo: Dioctophymatidae) en Hoplias malabaricus (Characiformes: Erythrinidae) en el Estado de Rondônia, Amazonía Occidental, Brasil

Dionatas Ulises de Oliveira Meneguetti

Departamento de Biologia, Faculdade de Educação e Meio Ambiente, Ariquemes, Rondônia, Brasil

Marcos Paulo de Oliveira Laray

Departamento de Saúde, Faculdade de Educação e Meio Ambiente, Ariquemes, Rondônia, Brasil
Luis Marcelo Aranha Camargo

Instituto de Ciências Biomédicas V, Universidade de São Paulo, Monte Negro, Rondônia, Brasil

Departamento de Medicina, Faculdade São Lucas, Porto Velho, Rondônia, Brasil

\section{RESUMO}

O presente artigo descreve o primeiro relato de larvas de Eustrongylides sp. em Hoplias malabaricus Bloch, 1794 (Characiformes: Erythrinidae) no Estado de Rondônia, Amazônia Ocidental, Brasil, sendo mais um parasita com a possibilidade de contaminar humanos no Estado.

Palavras-chave: Parasitologia; Nematoides; Traíra; Doenças dos Peixes.

\section{INTRODUÇÃO}

Todas as espécies de peixes podem abrigar uma ou mais espécies de parasitas, que podem ser encontradas tanto na fase adulta, quanto na fase larval. Eustrongylides sp. são nematoides encontrados em sua fase larval em algumas espécies de peixes carnívoros, como Auchenipterus nigripinnis, Brycon hilarii, Galeocharax humeralis, H. malabaricus, Paratrygon sp., Pinirampus pirinampu, Poeciliidae sp., Pseudoplatystoma corruscans, Pseudoplatystoma fasciatum, Rhaphiodon vulpinus, Salminus maxillosus, Serrasalmus nattereri, Synbranchus marmoratus' e Cichla ocellaris ${ }^{2}$. O parasita adulto é encontrado no esôfago, no pró-ventrículo e no intestino de aves aquáticas piscívoras, e o homem pode ser infectado acidentalmente ao se alimentar de peixe cru ou mal passado ${ }^{3}$.

H. malabaricus, conhecida popularmente como traíra, é um peixe carnívoro e atua como importante hospedeiro definitivo, intermediário e paratênico de helmintos, com destaque para larvas de nematoides.

\footnotetext{
Correspondência / Correspondence / Correspondencia:

Dionatas Ulises de Oliveira Meneguetti

Avenida Machadinho 4349. Bairro: Setor 6

CEP: 76870-293 Ariquemes-Rondônia-Brasil

Tel.: +55 (69) 3536-6600

E-mail: dionatas@icbusp.org
}

Essa espécie apresenta ampla distribuição na América do Sul, sendo encontrada em ambientes dulcícolas. Tem hábito preferencialmente noturno, é resistente a baixas concentrações de oxigênio na água e é utilizada como fonte alimentar por aves piscívoras e também por mamíferos, incluindo o homem 4 .

O presente estudo objetivou relatar, pela primeira vez, larvas de Eustrongylides sp. em $\mathrm{H}$. malabaricus, no Estado de Rondônia, Amazônia Ocidental.

\section{MATERIAIS E MÉTODOS}

Foram analisados 30 espécimes de $H$. malabaricus, (Licença Permanente IBAMA, número: 14934-1), com variação de peso entre $300 \mathrm{~g}$ a $800 \mathrm{~g}$ e comprimento total variando de $20 \mathrm{~cm}$ a $35 \mathrm{~cm}$, pescados com linha e anzol em um córrego ( $10^{\circ} 23^{\prime} 46^{\prime \prime} \mathrm{S}$ e $63^{\circ} 51^{\prime}$ '52" W), localizado no Município de Buritis, Rondônia, durante os meses de julho e agosto de 2012, e encaminhados para - laboratório de parasitologia da Faculdade de Educação e Meio Ambiente (FAEMA), localizada no Município de Ariquemes, Rondônia, onde foram realizadas as análises parasitológicas. A classificação taxonômica dos parasitas seguiu padrão descrito por Vicente e Pinto'; e Rêgo e Vicente ${ }^{5}$, por meio de características morfológicas visualizadas em microscopia óptica, com aumento de 40x (Figura 1). 

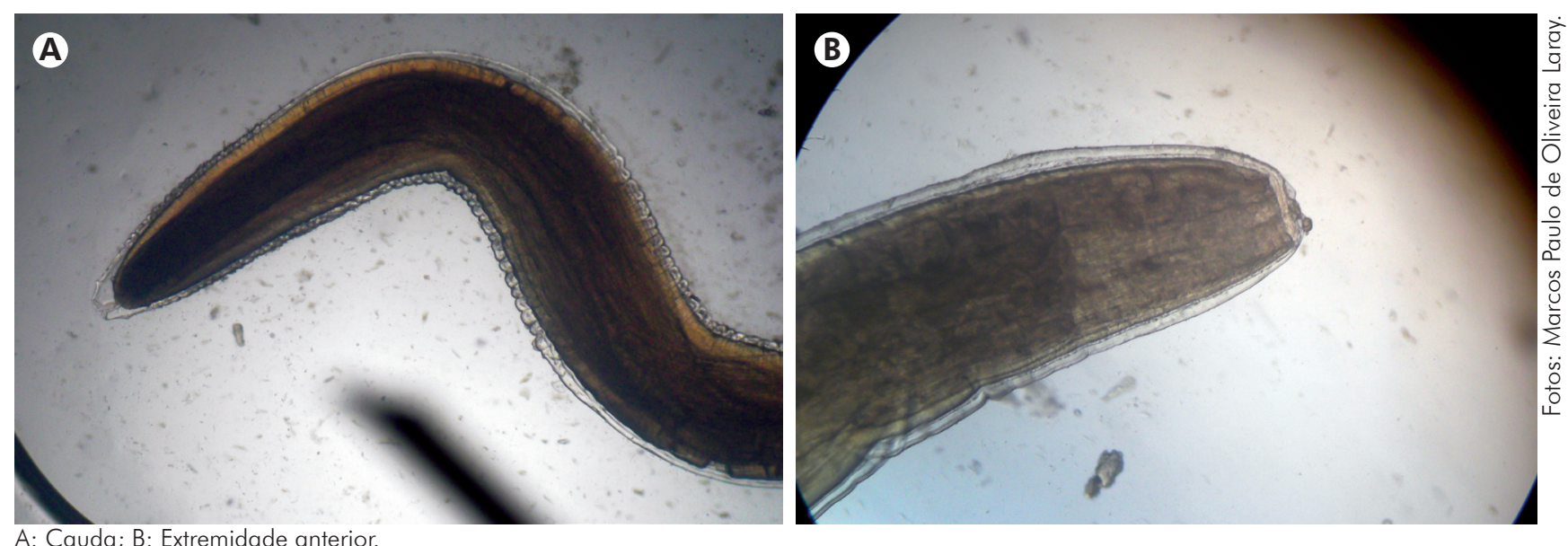

Figura 1 - Larvas de Eustrongylides sp. (aumento de 40x)

\section{RESULTADOS}

Dos 30 peixes analisados, 28 (93,3\%) estavam parasitados. Em 100\% dos casos foram encontradas unicamente larvas de Eustrongylides sp., e todas na musculatura esquelética do hospedeiro (Figuras 2A, 2B e
2C). Foram coletados 44 espécimes de Eustrongylides sp., com intensidade média de 1,47 por $H$. malabaricus analisada. As larvas de Eustrongylides sp. encontradas correspondem ao terceiro estágio larval ${ }^{6}$, com tamanho variando de $5 \mathrm{~cm}$ a $7 \mathrm{~cm}$ (Figura 2D).

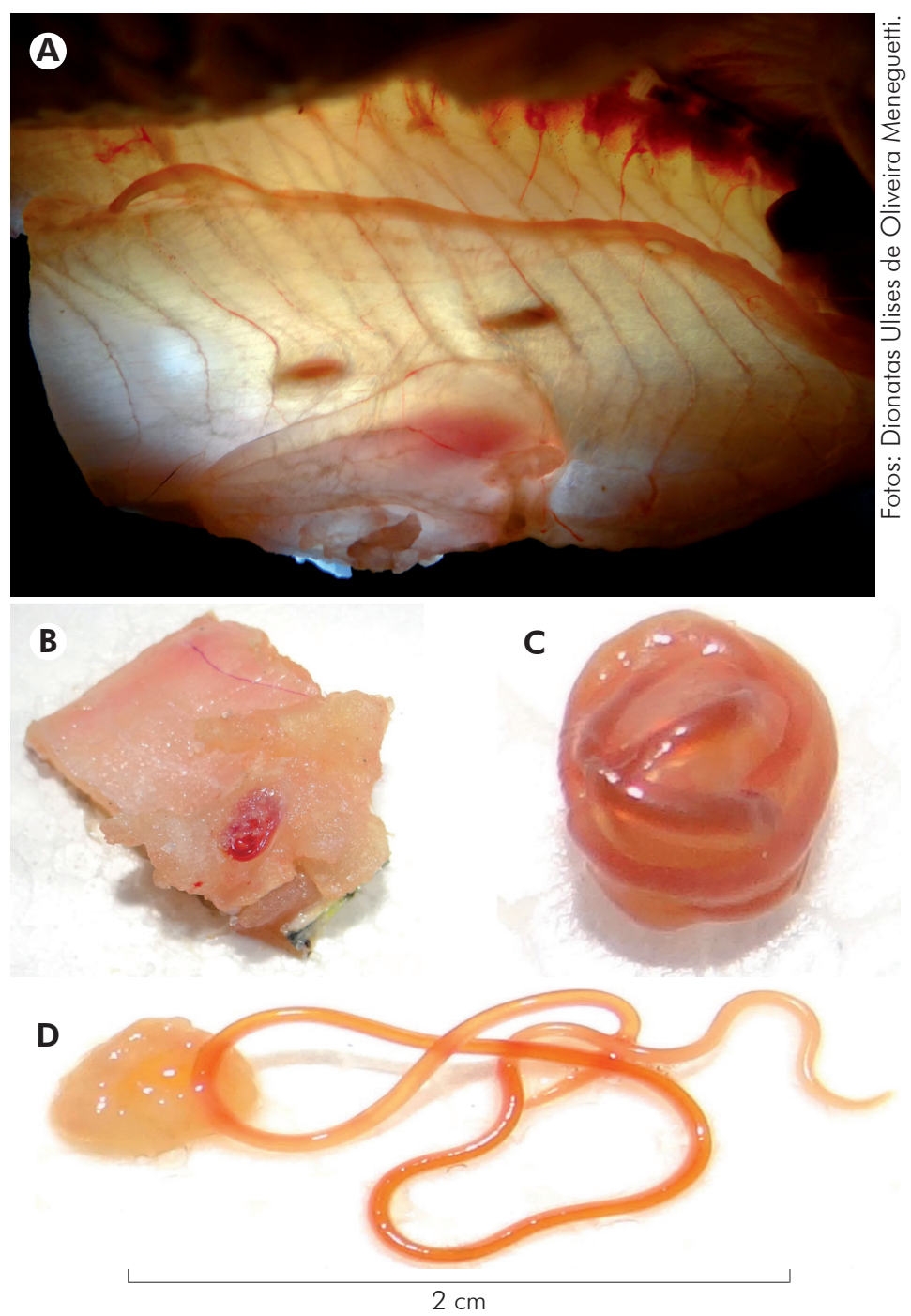

A e B: Cistos de Eustrongylides sp. na musculatura esquelética de $H$. malabaricus; C: Cistos de Eustrongylides sp. isolados da musculatura esquelética de H. malabaricus; D: Cisto eclodido e larva de Eustrongylides sp.

Figura 2 - Larvas de Eustrongylides sp. 


\section{DISCUSSÃO}

Apesar de, no Brasil, não haver registros de parasitismo em humanos por Eustrongylides sp., essa hipótese não pode ser descartada, principalmente na Região Amazônica, onde é comum as crianças ingerirem pequenos peixes crus com a crença de que com isso aprendem a nadar. Caso semelhante ocorreu em Nova Jersey, nos Estados Unidos, onde dois parasitas vivos foram removidos a partir da cavidade peritoneal de um jovem de 17 anos de idade, que se queixava de dor abdominal intensa no quadrante inferior direito, onde foi observada perfuração intestinal. Os vermes mediam $55 \mathrm{~mm}$ e $59 \mathrm{~mm}$ de comprimento e foram identificados como larvas de quarta fase de Eustrongylides sp. $\bigcirc$ jovem relatou que tinha o costume de engolir pequenos peixes vivos enquanto pescava ${ }^{7}$. Relato similar foi registrado por outro paciente, após a remoção cirúrgica de Eustrongylides sp. do seu intestino ${ }^{8}$.

sintoma de perfuração intestinal ocasionado por Eustrongylides sp. em humanos já havia sido descrito em 1982 pelo Center for Disease Control dos Estados Unidos da América9. Essas perfurações foram confirmadas experimentalmente em coelhos infectados com larvas de Eustrongylides sp. que apresentaram lesões gástricas por congestão e morte por peritonite $\mathrm{e}^{10,11}$.
Outra forma de infecção por esse parasito é a ingestão de peixe cru (sashimi) contaminado ${ }^{12}$. O sashimi vem sendo também preparado com peixes regionais (informação pessoal), dentre eles duas espécies Serrasalmus nattereri e Cichla ocellaris, nos quais já foi confirmada contaminação por larvas de Eustrongylides sp. ${ }^{7}$.

\section{CONCLUSÃO}

A ocorrência de larvas de Eustrongylides sp. em H. malabaricus é registrada em várias regiões brasileiras: Sul (Rio Grande do Sul13 e Paraná ${ }^{2}$ ), Sudeste (Espírito Santo $^{3}$ ), Centro-Oeste (Mato Grosso ${ }^{4}$ ) e Norte (Pará ${ }^{4}$ ). Este é o primeiro relato de Eustrongylides sp. ocorrente no Estado de Rondônia, sendo mais um parasita com probabilidade de infecção em humanos.

\section{AGRADECIMENTOS}

Aos professores dr. Daniel Moura de Aguiar e dr. Edivaldo Sampaio de Almeida Filho da Universidade Federal de Mato Grosso, e dr. Sergio Carmona de São Clemente da Universidade Federal Fluminense, pela colaboração na identificação do parasita. Aos professores ms. Leandro José Ramos e ms. Ranto André Zan da FAEMA, pela colaboração na coleta de campo e análise parasitológica.

\section{First report of Eustrongylides sp. larvae (Nematoda: Dioctophymatidae) in Hoplias malabaricus (Characiformes: Erythrinidae) in Rondônia State, Western Amazon, Brazil}

\section{ABSTRACT}

This paper describes the first report of Eustrongylides sp. larvae in Hoplias malabaricus Bloch, 1794 (Characiformes: Erythrinidae) in Rondônia State, Western Amazon, Brazil, and it is more a parasite which can infect humans in that area.

Keywords: Parasitology; Nematoda; Trahiras Fish; Diseases.

\section{Primer relato de larvas de Eustrongylides sp. (Nematodo: Dioctophymatidae) en Hoplias malabaricus (Characiformes: Erythrinidae) en el Estado de Rondônia, Amazonía Occidental, Brasil}

\section{RESUMEN}

El presente artículo describe el primer relato de larvas de Eustrongylides sp. en Hoplias malabaricus Bloch, 1794 (Characiformes: Erythrinidae) en el Estado de Rondônia, Amazonía Occidental, Brasil, tratándose de un parásito más con la posibilidad de contaminar humanos en el Estado.

Palabras clave: Parasitología; Nematodos; Trahiras; Enfermedades de los Peces.

\section{REFERÊNCIAS}

1 Vicente JJ, Pinto RM. Nematóides do Brasil. Nematóides de peixes. Atualização: 1985-1998. Rev Bras Zool. 1999 set;16(3):561-610.
2 Martins ML, Santos RS, Marengoni NG, Takahashi HK, Onaka EM. Seasonality of Eustrongylides sp. (Nematoda: Dioctophymatidae) larvae in fishes from Paraná river, South-Western Brazil. B Inst Pesca. $2009 ; 35(1): 29-37$. 
3 Caetano MS, Senra LB, Starling AG, Santos GM, Gonçalves Júnior LP, Amaral AA. Ocorrência de Eustrongylides sp. em traíras (Hoplias malabaricus) do córrego do capim, afluente do rio Norte Alegre - ES. XV Encontro Latino Americano de Iniciação Científica e XI Encontro Latino Americano de Pós-Graduação Universidade do Vale do Paraíba; 2011.

4 Barros LA, Moraes Filho J, Oliveira RL. Larvas de nematóides de importância zoonótica encontradas em traíras (Hoplias malabaricus Bloch, 1794) no município de Santo Antonio do Leverger, MT. Arq Bras Med Vet Zootec. 2007;59(2):533-5.

5 Rêgo AA, Vicente JJ. Eustrongylides ignolus Jagerskiold, 1909 (Nematoda: Dioctophymatoidea), parasito de peixes, anfíbios, répteis e aves. Distribuição e taxonomia. Cienc Cult. 1988;40(5):478-83.

6 Friend M, Franson JC. Field manual of wildlife diseases: general field procedures and diseases of birds. Biological Resources Division; 1999. (Information and Technology Report; 1999).

7 Eberhard ML, Hurwitz H, Son AM, Coletta D. Intestinal perforation caused by larval Eustrongylides (Nematoda: Dioctoplymatoidae) in New Jersey. Am J Trop Med Hyg. 1989 Jun;40(6):648-50.

8 Narr LL, O'Donnell JG, Libster B, Alessi P, Abraham D. Eustrongylidiasis: a parasitic infection acquired by eating live minnows. J Am Osteopath Assoc. 1996 Jul;96(7):400-2.
9 Centers for Disease Control and Prevention. Intestinal perforation caused by larval Eustrongylides - Maryland. Morb Mortal Wkly Rep. 1982 Jul;31 (28):383-9.

10 Shirazian D, Schiller EL, Glaser CA, Vonderfecht SL. Pathology of larval Eustrongylides in rabbit. J Parasitol. 1984 Oct;70(5):803-6.

11 Barros LA, Tortelly R, Pinto RM, Gomes DC. Effects of experimental infections with larvae of Eustrongylides Ignotus Jäegerskiold, 1909 and Contracaecum multipapillatum (Drasche, 1882) Baylis, 1920 in rabbits. Arq Bras Med Vet Zootec. 2004 Jun;56(3):325-32.

12 Wittner M, Turner JW, Jacquette G, Ash LR, Salgo MP, Tanowitz HB. Eustrongylidiasis: a parasitic infection acquired by eating sushi. N Engl J Med. 1989 Apr;27(17):1 124-6.

13 Rodrigues AP, Pesenti TC, Berne MEA. Nematódeos parasitos de Hoplias malabaricus comercializados em Pelotas, Rio Grande do Sul. XVIII Congresso de Iniciação Científica e XI Encontro de Pós-Graduação. Universidade Federal de Pelotas; 2009.

14 Benigno RNM, São Clemente SC, Matos ER, Pinto RM, Gomes DC, Knoff M. Nematodes in Hoplerytrinus unitaeniatus, Hoplias malabaricus and Pygocentrus nattereri (pisces characiformes) in Marajó Island, Brazil. Rev Bras Parasitol Vet. 2012 Apr-Jun;21 (2): 165-70. 